The "subtle violence"

Adultized children and educational challenges

La "violenza sottile"

Infanzia adultizzata e sfide pedagogiche

Silvia Demozzi ${ }^{1}$

\begin{abstract}
The article focuses on the topic of "adultized" children seen as an expression of a "subtle violence" that Western society plays against new generations. After defining the concept of subtle violence, the article analyses those situations in which childhood risks its disappearance and in which children are treated as "little adults". What are the challenges this trend poses to the pedagogical thinking? And which are the trajectories that, starting from both micro and macro analysis, can be pursued so that adult society can guarantee the protection and respect of children rights, first of all, the right to have a childhood? Conclusions try to answer - albeit not exhaustively - to these questions, outlining some epistemological paths in order to overstep the great paradox of a "dumb childhood" and for the recognition of its right to be respectfully cared.
\end{abstract}

Keywords: childhood, adultized children, violence, children rights, education.

\title{
Prima, l'infanzia
}

"Prima l'infanzia", scrive Rilke e poi, d'improvviso, "il terrore, scuola, barriere..." e una "perdita profonda" (1923); lo scrive da un letto d'ospedale, ricoverato per un male oscuro, e compone questa "biografia immaginaria" con cui spalanca le porte al ricordo del Rilke-bambino. Tra le sue parole, un misto di nostalgia per un tempo prezioso, quel "prima" in cui tutto pare muoversi all'insegna della gioia e dell'onnipotenza, ma che, presto, e per di più all'improvviso, si trasforma in "terrore", nella perdita - profonda - di un'età che non fa ritorno se non con il gusto amaro di una rievocazione ormai lontana. Basterebbero queste parole a testimoniare l'importanza, da parte di chi ha a cuore l'infanzia, di proteggere e tutelare quel "prima" così ricco di significato per la vita di ciascun bambino e bambina. Ma, come ben sappiamo, nonostante

\footnotetext{
${ }^{1}$ Università di Bologna.
} 
i grandi traguardi raggiunti - sia in termini di conoscenza sia legislativi - pare che per garantire all'infanzia - nella sua accezione socio-educativa - almeno la sua sopravvivenza, si debbano compiere degli sforzi le cui ricadute, in troppi contesti, non sono ancora realtà. Come ci ricorda Contini, in ogni tempo «le condizioni di vita dei soggetti socialmente più deboli in termini di potere»-e l'infanzia (in-fans), senza parola, rientra tra questi - «dipendono totalmente dai voleri dei soggetti più forti e dal riconoscimento o meno, da parte di questi, dei loro diritti» (Contini, 2016, p. 17).

Ripercorrendo a ritroso la storia dell'infanzia - categoria, come ci ricorda Postman (2005), che compare e scompare nel tempo - non è difficile rintracciare indizi di continue violazioni di quelli che nel 1989 sono stati decretati, con la Convenzione Onu sui diritti dell'infanzia (CRC), i diritti inviolabili di ogni bambino, proprio in virtù della sua appartenenza a una condizione - quella infantile - portatrice di bisogni specifici e particolari. Con la CRC, infatti, seppur molto tardi in termini di storia dell'umanità, la società adulta riconosce, per la prima volta, i bambini e le bambine come cittadini portatori individuali dei loro diritti, il primo tra tutti, quello di avere un'infanzia. Ma la stessa società adulta, che ha riconosciuto e siglato nero su bianco i diritti dei bambini, pare averli misconosciuti nel tempo, spingendosi oltre ogni limite e non arrestandosi, in taluni casi (quelli più terribili e disumani), nemmeno di fronte al loro diritto alla vita (Contini, 2016).

Da sempre, in ogni tempo e con ogni forma, purtroppo, l'infanzia è stata vittima - spesso inconsapevole e muta - di una violenza da parte degli adulti (Cambi, Ulivieri, 1990), ora avallata omertosamente, ora denunciata senza conseguenze, ora sottaciuta con complicità... Dal microcosmo familiare al macro contesto globale, manifestazioni di "pedagogia nera" (Miller, 2010) si sono susseguite nel tempo, passando per la violenza più efferata (fino all'epoca tardo romana l'infanticidio non era considerato un reato), attraverso forme di conclamata promiscuità (pensiamo al Medioevo in cui, a 7 anni, un bambino era considerato a tutti gli effetti un adulto), fino ad arrivare a forme più sottili, meno fisiche, improntate all'umiliazione e alla mortificazione. Così l'infanzia, detta da altri e mai da sé, non si vede riconosciuto null'altro se non il suo "non essere ancora" e questa assenza, insita sin nella sua stessa etimologia, descrive qualcosa di "mancante" e "incompiuto", un'alterità che da sempre interroga e, al contempo, spaventa. L'infanzia è "minore" rispetto a qualcosa d'altro e rispetto, soprattutto, a qualcun altro - l'adulto - che ne descrive $\mathrm{i}$ tratti e ne delinea i percorsi per portarla ad uscire da questa sua "alterità" (Becchi, 1982). Ed è forse proprio a partire da questa impossibilità di dirsi, di definirsi e di replicare, che prende corpo il rischio di questa eterna scomparsa e si concretizza la grande difficoltà, per l'umanità adulta, di prendersi cura autenticamente di quel "prima" così prezioso e delicato (Demozzi, 2016). 


\section{La "violenza sottile": quando l'infanzia è adultizzata}

E se l'idea d'infanzia o, per dirla con Ariés (1994), il suo "sentimento", ha dovuto lottare nel tempo per affermarsi e diffondersi in tutti i contesti sociali e, non solo - quindi - in quelli privilegiati, non possiamo non riconoscere come essa veda costantemente minacciata la sua sopravvivenza e come l'umanità adulta, ancora oggi e anche qui, nel più "avanzato" Occidente, faccia molta fatica a prendersene cura. Ciò è certamente più evidente nei contesti di assoluta povertà e svantaggio socio-educativo, ma a ben vedere, purtroppo, "schegge di pedagogia nera" (Demozzi, 2016) permangono ancora, più o meno consapevolmente, sia in alcune pratiche sia in una più generalizzata tendenza, ben descritta da Contini (2010) con il concetto di "adultizzazione".

Di fronte all'incuria, all'indifferenza e alla crudeltà che tanti - troppi bambini del mondo ogni giorno subiscono per ragioni così lontane dal e in opposizione al diritto alla cura, le "nostre" infanzie appaiono (e sono) privilegiate (sebbene di privilegio non si dovrebbe nemmeno parlare, poiché ci stiamo muovendo nel campo dei diritti e, in primis, dei diritti umani): "i nostri bambini stanno bene" - recita il video-documentario "Corpi bambini. Sprechi di Infanzie" (IbcMovie, 20132) - sono curati, ben nutriti, frequentano scuole e servizi educativi di qualità...

I "nostri" bambini, in effetti, sono bambini la cui infanzia "parte" da una "condizione-data" certamente immune dalle tante nefandezze e complicazioni, nonché dalle miriadi di variabili nefaste che influenzano la vita in molti contesti del pianeta; sono infanzie, le "nostre", che hanno tutte le carte in regola (non solo, quindi, quelle più astratte delle Convenzioni) per vedersi garantito un certo benessere. Nel procedere con questa separazione (i nostri/gli altri), ammettiamo di compiere una "selezione" di non poco conto che, per dirla con Contini, «lascia in ombra un numero molto elevato di piccoli, rimuovendo il tema-problema dei diritti» (2016, p. 30); è dunque un'operazione impropria (e eticamente discutibile), ma che ora, nella sede di questo saggio, ci serve per accendere un riflettore su un'altra questione - certamente meno allarmante che ci riguarda da molto vicino.

Il fenomeno cui stiamo assistendo porta con sé un corollario pesante in termini educativi, ovverosia quello di vedere misconosciuta, ancora una volta, la categoria infanzia: bambine e bambini, infatti, subiscono sempre più spesso un processo di adultizzazione della loro infanzia, in cui "cade" la separazione $\mathrm{o}$, per dirla in altre parole, la necessaria a-simmetria che dovrebbe garantire un accesso graduale al mondo adulto e, soprattutto, tutelare una cura educativa rispettosa dei bisogni di questa età.

\footnotetext{
${ }^{2}$ Il documentario è visionabile alla pagina web www.corpibambini.wordpress.com.
} 
L'infanzia dell'attualità è abitata sempre più da "piccoli adulti", competenti, capaci, smaliziati; il prodotto, molto ben riuscito, di una società caratterizzata da forte competitività, individualismo, crisi dei valori, appiattimento culturale, corsa al successo e all'arricchimento (Demozzi, 2016, p. 54).

Il processo di adultizzazione dell'infanzia, però, non può essere definito (e nemmeno analizzato) senza fare riferimento al suo contraltare, ovverosia alla generalizzata tendenza all'"infantilizzazione" da parte del mondo adulto. Gli adulti, infatti, coloro che, essendo più grandi, come tali dovrebbero comportarsi, molto spesso subiscono - poiché questo è il paradigma sociale dominante - il fascino dell' "eterna giovinezza", ne rincorrono il mito (certamente aiutati dai media e dal mercato che costantemente offrono i mezzi e gli strumenti), incapaci poi, nella grande sfida del "divenire se stessi" (Ehrenberg, 2010), di assumersi le responsabilità richieste e di districarsi tra insicurezze e fragilità, anche nella cura dei più piccoli (Ammaniti, 2015). E così i più piccoli, a cui spesso viene richiesto di scegliere e di decidere per conto loro, finiscono per passare, in un sol colpo, da "principi" a "tiranni" che, sapientemente, si insinuano nelle crepe scricchiolanti della "regia adulta" e ne minacciano la sempre più fragile autorevolezza ${ }^{3}$.

Quanto giova questo "surplus di mondo adulto" ai bambini? Quanto, questo loro essere "bambini tiranni", "bambini capolavori", "bambini trofeo", "bambini partner", "bambini danzatori", "bambini trilingue", "bambini cantanti", "bambini calciatori"... giova alla loro infanzia? Cosa significa vivere un'infanzia da bambini "che sono un po' meno bambini"? Quando potranno tornare ad essere (ammesso che lo siano mai stati), per dirla con Bernardini (2012), "bambini e basta"?

Da qui parte e continua la nostra riflessione sulla "violenza sottile" nei confronti della "nostra" infanzia, una violenza che, più o meno limpidamente, si accompagna al fenomeno dell'adultizzazione, soprattutto quando, conseguentemente alla caduta dell'a-simmetria, i bambini e le bambine vengono travolti da messaggi, immagini e parole di cui sanno tutto ma, al contempo, non capiscono niente, come afferma Galimberti (2002), perché "analfabeti delle emozioni".

\section{Dove si nasconde la "violenza sottile"?}

E allora vediamo dove si annida la "violenza sottile", in quali pieghe della nostra quotidianità, più o meno diffusamente - in base ai contesti e alle

${ }^{3}$ Sulla questione autorità vs autorevolezza o, riprendendo le parole della stessa autrice, "dall'autoritarismo alla democraticità", si rimanda al testo di A. Gigli (2016, pp. 36 e seguenti). 
situazioni -, questa violenza si insinua senza fare rumore tanto che il mondo adulto, abbagliato dalla luce di ciò che è più trasparente, non si accorge operando una pesante rimozione - delle ombre che minacciano l'infanzia nella sua forma più autentica e, per usare una aggettivazione di stampo problematicista, "inattuale" (Bertin, 1977; Demozzi, 2016).

Dietro la narrazione di un'infanzia felice e spensierata si nascondono molte contraddizioni: sono, infatti, i valori che dominano una società (denaro, potere, successo) a condizionare le pratiche di cura e l'atteggiamento (dis)educante nei confronti dell'infanzia. Essere "bambini e basta" non è sufficiente in una parte di mondo che anela all'arricchimento e ai risultati, che muove e si muove esclusivamente su spinte individualiste e consumiste, un mondo in cui le leggi - anche quelle (a)morali - le fanno i mercati che hanno individuato, proprio nell'infanzia, uno degli interlocutori privilegiati.

Partendo dai diritti riconosciuti dalla CRC, portiamo la nostra riflessione su une delle dimensioni più significative per lo sviluppo infantile e - come ci insegnano gli antropologi e gli psicologi - per lo sviluppo umano in generale: il gioco. Il gioco è universale e appartiene, di diritto appunto, alla "sanità" dell'infanzia, una delle prime forme di comunicazione, il linguaggio privilegiato di tutte le bambine e i bambini del mondo, la palestra protetta in cui fare esperienza del Sé in relazione al mondo (Winnicott, 1971).

Scrive Dolto che si deve lasciare che il bambino «eserciti liberamente il corpo: rotolare, strisciare, inarcarsi, alzarsi con appoggio, ecc.» e aggiunge che «prima o poi il bambino si farà male (...)» (2005, p. 67). Bastano queste poche frasi e il tutto appare alquanto anacronistico: sappiamo che i nostri bambini appartengono alla prima generazione "senza ginocchia sbucciate", conosciamo bene i contesti di gioco e, nonostante la resistenza di alcune buone eccezioni, sappiamo anche che la dimensione ludica, oggi, è molto strutturata, controllata, raramente lasciata al "caso" o, per meglio dire, alla regia esplorativa e curiosa di bambini e bambine.

Ma, si potrebbe obiettare che in termini di contesti e, ancora di più di oggetti, ai nostri bambini non manca nulla; ed in parte è vero. Tuttavia, sappiamo anche che in Italia la crisi economica ha colpito pesantemente $\mathrm{e}$, nei fatti, anche i servizi educativi e, in generale il terzo settore, hanno subito ingenti ridimensionamenti che producono, tra le loro ricadute, un impoverimento dell'offerta educativa (in particolare, in alcune zone del paese) anche in termini di spazi per il gioco e per l'attività motoria. Quanto agli oggetti, è vero, i giocattoli non mancano; anzi, si potrebbe azzardare, che la "troppa cura", istigata dall'azione del mercato, fa sì che i nostri bambini siano "sommersi" dagli oggetti, in mezzo ai quali tendono a perdersi e a scomparire e, cosa ancora più grave e violenta, non conoscono la pazienza dell'attesa, il valore del tempo che separa un desiderio dalla sua realizzazione, poiché tutto è sùbito e non c'è proprio lo spazio per immaginare, sognare o fantasticare. 
Quanta differenza tra un desiderio che si affaccia, cresce, matura, sa attendere e una voglia che irrompe, si impone, reclama l'immediato soddisfacimento: accogliere e corrispondere a quest'ultima vuol dire impoverire il "bagaglio" di emozioni dei bambini e porre un'ipoteca sulla loro possibilità di interagire positivamente con le domande della vita e nei rapporti con gli altri, empatizzando con loro e decentrandosi da se stessi (Contini, 2016, p. 34).

Sostiene la sociologa D'Amato (2014) che oggi possiamo definire il bambino come "puer oeconomicus", perfettamente in linea, dunque, con i canoni della società attuale: fin dalla più tenera età il bambino è indotto a scambiare i suoi desideri per bisogni, indotto dai mercati e dalle multinazionali (del giocattolo, del cibo-spazzatura, del video-gioco) che trovano il loro terreno più fertile proprio nelle menti più recettive e plasmabili (Oliverio Ferraris, 2008).

Il gioco, dunque, da arena privilegiata per lo sviluppo psico-fisico del bambino rischia, oggi, di essere identificato con gli oggetti, e, in particolare, con gli oggetti che si possiedono o che si desidera possedere. È questa una forma di "violenza sottile" con la quale la società adulta sottrae all'infanzia una delle sue dimensioni più preziose, ovverosia, per dirla con Bateson (1977), quella "cornice" entro cui sperimentare l'immaginazione e l'azione, e, al contempo, imparare a comunicare, a gestire le frustrazioni, a relazionarsi col mondo.

Un'altra dimensione molto frequentata dall'infanzia in cui si concretizza la minaccia alla sua stessa sopravvivenza è quella dello sport e, in generale, dell'attività motoria. Fin da molto presto "il bambino-adulto" prende parte a qualche attività extra scolastica (musica, sport, lingua straniera) riempendo la "sua" agenda in ogni pagina, non lasciando spazio, così, a tempi vuoti o al sentimento di noia, tanto temuto da mamma e papà. Nello scegliere una attività sportiva per i loro figli, i genitori di oggi compiono una azione ben informata e consapevole, fondata sul riconoscimento del valore dell'attività motoria nello sviluppo psico-fisico dei bambini e nel rafforzamento delle sue competenze socio-relazionali (Morandi, 2016). Tuttavia, anche il contesto sportivo frequentato dai piccolissimi rischia, soprattutto nelle società più affermate e organizzate, di divenire terreno per l'insediarsi di una "violenza sottile". Parole come "prima squadra", "convocazioni", "qualificazioni", "performance"... dominano certi ambienti e prevaricano sulle più educative "gioco", "sfida", "cooperazione", "partecipazione"; l'attenzione per risultato sportivo, in termini di vittorie e affermazioni, diviene dominante, ma ciò è in linea - come abbiamo visto - con la cornice più grande all'interno della quale si collocano le esistenze quotidiane dei micro-contesti che, inevitabilmente, subiscono i condizionamenti delle tendenze globali. E, dunque, assistiamo ad adulti (genitori e allenatori) che proiettano le loro aspirazioni agonistiche sui 
più piccoli, essendo disposti a tutto pur di vederli divenire "campioni"; ma, allo stesso tempo, raccogliamo i lamenti, da un lato, di tanti allenatori che sempre più faticano a relazionarsi con la categoria di "genitori ultras" e, dall'altro, di tanti genitori che, a malincuore, ritirano i propri figli dalle attività sportive poiché consapevoli della nocività dello stress della competizione, dei ritmi serrati e delle fatiche di coordinare l'incastro tra le tante attività.

Per alcuni che, nonostante le fatiche, diventano un giorno campioni, quanti sono i bambini, i "nostri", che si ritrovano a giocare una partita che non hanno scelto ma che, per paura di deludere i propri adulti di riferimento o, peggio ancora, di perdere il loro amore, non possono abbandonare? Possiamo parlare anche in questo caso di violenza? Potremmo, prendendo in prestito la definizione di Galtung (1990), parlare di "violenza culturale", non tanto praticata da terribili aguzzini, bensì quotidianamente da individui "normali", anche nella veste di genitori e insegnanti, quando accettano, spesso senza rendersene conto $\mathrm{o}$, comunque, non esercitando un pensiero critico, ciò che viene "suggerito" dallo spirito dei tempi e, di conseguenza, trasferito, sotto forma di saperi e valori, anche alle generazioni più piccole.

Il punto è che, purtroppo, controllare questo tipo di processo diventa sempre più difficile $\mathrm{e}$, nel terreno dell'attuale, nell'intreccio complesso delle dinamiche dei tanti contesti e dei tanti attori, i flussi di socializzazione sono «sempre più aperti ad attori esterni alla famiglia e si realizzano sempre più precocemente nella vita dei bambini, attraverso una infinità di canali comunicativi non facilmente controllabili e neutralizzabili» (Forni, 2005, p. 45), anche volendolo.

Ed è proprio attraverso questi stessi canali, quelli propri di una "società dell'informazione", che si realizza una ulteriore forma di "violenza sottile" - meno abitata in termini di numeri - ma molto visibile e diffusa a livello di percezione di massa. Attraverso un processo di "vetrinizzazione sociale", assistiamo ad una progressiva spettacolarizzazione di soggetti e contenuti nei principali ambiti delle società occidentali: gli affetti, la sessualità, il corpo, l'attività sportiva, i media, il tempo libero, i luoghi del consumo, gli spazi urbani e persino le pratiche relative alla morte (Codeluppi, 2007). Un processo, questo, fortemente rafforzato dai media che, attraverso il "linguaggio della vetrina", trasformano fenomeni collettivi ed individuali in "merce" da esposizione e a cui, difficilmente, i soggetti riescono a sottrarsi, anche e soprattutto i più piccoli.

E, dunque, assistiamo a una carrellata di piccole Lolite e piccoli gagà ammiccanti, a una schiera di "piccoli adulti in vetrina" che ci attraggono e ci stupiscono con i loro "talenti" e la loro straordinaria bellezza. Protagonisti di trasmissioni televisive (la cui audience è fatta da adulti) in prima serata o di campagne pubblicitarie sulle riviste di alta moda, piccoli e piccole spettacolarizzati, osannati, imbellettati fino al grottesco (con denti finti, 
parrucche, abbronzatura spray) si muovono come burattini con in bocca "parole adulte" che mai sono state censurate e di cui non conoscono il significato. Una vera e propria violenza, questa, che fa dell'infanzia uno strumento per rispondere al mito del successo e della popolarità veloce, e che sancisce una delle più grevi alterazioni del diritto a vivere e crescere in ambienti sani ed educativamente significativi.

La società dei mercati e dell'informazione ha forse sorpassato il grande limite che sanciva e garantiva un rispetto all'infanzia fatto anche di pudore e protezione; manca oggi una coscienza più grande, che responsabilmente protegga l'infanzia dal suo essere usata come merce tra le più pregiate e le meno costose. E l'infanzia dei palinsesti e delle copertine (ma anche delle sfilate o delle sagre di paese) diviene modello di identificazione per i suoi interlocutori che, da ignari spettatori, si ispirano alle tendenze dell'attuale aderendo così alle sue logiche, anche a quelle meno nobili e più interessate. Si tratta, ancora una volta, di una violenza che Bourdieu definisce "simbolica", veicolata soprattutto attraverso la televisione e le nuove tecnologie, quando ad esse non ci si approcci con senso critico e con la dovuta consapevolezza. È una violenza "perniciosa" che trae la sua forza a partire dalla complicità - anche inconsapevole - tra chi la compie e chi la subisce (Bourdieu, 1997, p. 17). Una violenza che va, prima di tutto, portata alla luce, poiché è sempre troppo grande la rimozione che la società adulta compie più o meno consapevolmente; è una violenza che va fermata, per opera di una riflessione e di una azione quotidiane, nei contesti più grandi, così come nei micro-spazi della quotidianità, ripartendo dai diritti e dalla necessità, sempre più urgente, di mettere in atto azioni di cura tese a tutelarli.

\section{Dalla consapevolezza alla responsabilità}

Dicevamo qualche paragrafo fa che la società adulta, oggi, è essa stessa vittima del predominare di alcuni valori dominanti, nonché di una crisi, non solo economico-sociale, che ha visto uno sgretolamento finanche delle "grandi narrazioni". I padri e le madri di oggi, così come la classe di educatori e insegnanti, rientrano a pieno titolo tra coloro che hanno vissuto un processo di socializzazione che - a cavallo tra i due secoli - si è visto caratterizzare per una serie di peculiarità: crisi del welfare-state e delle istituzioni tradizionali, affermazione di politiche neo-liberiste e trionfo del consumismo, radicamento dell'ideologia del "privato" e diffusione estrema dell'individualismo (Beck, 2000), nonché, per dirla con Bauman (2002), di "liquidità" lavorative ed esistenziali. L'infanzia di oggi, dunque, non può che "respirare" i prodotti di questo processo, assumendone, così, anche gli stessi valori. Il problema, però, è 
che in tal modo l'infanzia non vede protetti alcuni dei suoi diritti più specifici e rischia - come categoria sociale e culturale - di scomparire.

I valori che vengono trasmessi oggi ai bambini (...) rispecchiano fedelmente il disorientamento di quella generazione di adulti, la loro perdita di identità sociale, la loro incapacità di collocarsi in una dimensione temporale diacronica, con lo smarrimento del senso della storia e la mancanza di visioni per il futuro (Forni, ibid., p. 94).

Ci ritroviamo, quindi, di fronte ad un rischio doppio: per l'infanzia e per i suoi genitori. Individui, gli uni e gli altri, certamente più liberi, con un bagaglio inesauribile di informazioni, ma anche molti più fragili sul piano della sicurezza di sé e della gestione delle relazioni. Uscendo, quindi, da una facile logica accusatoria nei confronti della generazione dei "padri" e dei "maestri", riportiamo invece l'attenzione sulle premesse a livello globale da cui discendono poi, inevitabilmente, anche certe scelte e certi stili (dis)educativi.

La fragilità di questo tempo necessita di una "rivoluzione del pensiero" (Morin, 2000), una vera e propria messa in discussione di alcuni paradigmi socio-culturali ed economici che hanno messo a rischio la sopravvivenza (anche quella biologica) di tutti gli esseri viventi, di tutte le categorie e, quindi, della stessa infanzia. L'urgenza di un impegno etico e di una pedagogia che si facciano baluardo dei diritti dell'infanzia è la strada, forse, per ripartire e per prefigurare altri orizzonti, una strada che porterebbe con sé, molto probabilmente, vantaggi a livello sociale per l'intera umanità. Ripartire dall'infanzia, infatti, avendo a cuore la cura e la tutela dei diritti dei bambini, potrebbe essere una strada - seppur a rischio di fare una affermazione retorica - verso il recupero di nuovi valori, quali quelli della gratuità e della solidarietà, della speranza e dell'entusiasmo.

I bambini e le bambine non vanno, dunque, adultizzati nei consumi, negli atteggiamenti, nelle aspettative, ma va loro riconosciuto, riprendendo le parole del maestro Lorenzoni (2014), il loro potere di "pensare grande", intendendo con ciò un riconoscimento del valore esteso che le loro domande portano con sé. Uno dei punti più alti della $\mathrm{CRC}$ dell'89 è rappresentato dal rispetto delle idee dei bambini e dal loro diritto di poterle esprimere, tuttavia sappiamo bene che con l'equivalenza "corpo piccolo = pensiero piccolo" spesso finiamo per misconoscere la conquista di questa nobile tappa legislativa. Come afferma Lorenzoni nella bellissima narrazione della sua "avventura pedagogica":

i bambini devono essere ascoltati, perché di fronte al bello, alle difficoltà e anche alle tragedie della vita, sono capaci di nitidezza ed autenticità rare, che credo faccia bene a tutti incontrare (ibid., p. 13). 
Allora la pedagogia, tra le discipline in prima linea e come esempio, ma anche la cultura in generale potrebbero fare uno sforzo di umiltà e di "regressione", abbandonando l'urgenza di offrire risposte e, piuttosto, accogliendo e avendo a cuore (riprendendo l'I care di don Lorenzo Milani) le domande dell'infanzia, gli interrogativi che essa pone alla società adulta nel suo tentativo di crescita all'interno di essa. È questa, tra le tante valide proposte, quella che viene, ad esempio, dalla "filosofia dell'infanzia" (Kohan, 2014), secondo cui, proprio a partire dall'inquiry del filoso-fare, gli adulti possono accompagnare i bambini e le bambine nella costruzione di un loro pensiero critico e creativo e, al contempo, apprendere, a loro volta, dalle loro domande e riflessioni. L'infanzia diviene, così, "maestra" degli adulti nell'insegnare loro a recuperare le domande, a non accontentarsi di fronte alle prime risposte, in altre parole, a indagare la realtà. La proposta è quella di valorizzare e preservare il "tempo dell'infanzia", un tempo che ha molte affinità con la creatività e la ricerca filosofica, che affonda le radici nelle dimensioni della meraviglia e dello stupore (Lipman, 2001). Un'educazione che abbia a cuore il recupero del "tempo dell'infanzia" non è tanto una educazione che mira solamente a "istruire e formare" l'infanzia (dimensioni, intendiamoci bene, altrettanto importanti e da perseguire), bensì è un'educazione che si interroga su quali siano i pensieri e le pratiche migliori per essere coerenti con essa. Su quali siano le forme migliori per farlo, da tempo la ricerca e la riflessione delle scienze dell'educazione cercano di interrogarsi, scontrandosi costantemente con le difficoltà (e i paradossi) legate al dare voce a una categoria sociale che, di fatto, è da sempre considerata "muta". Ma i bambini e le bambine devono essere tutelati nel loro essere attori con diritto (anche di parola) all'interno della loro cultura e, come tali, vanno valorizzati e, in primis, ascoltati. Ł̀ quanto afferma Satta (2012) nella sua descrizione delle "culture dei bambini", quelle che essi, non solo subiscono ma che, al contempo, in qualità di soggetti dotati di intenzionalità, contribuiscono a trasformare. I bambini, continua Satta, "s'inventano l'infanzia" (ibid.), ovverosia interagiscono con gli adulti e prendono parte all'indispensabile processo di significazione della loro realtà, ciò che spesso manca, aggiungiamo noi, è il riconoscimento e la tutela da parte dei "grandi" di uno spazio e di un tempo in cui l'infanzia sia legittimata a compiere quest'invenzione. La sfida dei diritti, soprattutto laddove prendono piede e attutiscono certe forme di "violenza sottile", è anche questa: far sì che bambini e bambine abbiano a disposizione quei luoghi e quei momenti necessari ed adeguati affinché si approprino autonomamente del loro mondo, vivendolo alla loro altezza e con i tempi della loro maturazione.

Per dirla in altre parole, si potrebbero percorrere, anche nelle pratiche, quelle strade che promuovono l'ascolto dei bambini e ne valorizzano le idee (un esempio è rappresentato dalla Philosphy for Children), privilegiando la 
dimensione del dialogo e della discussione, in cui sin da piccoli si possa imparare il confronto e finanche il conflitto, non tanto per «vincere battaglie di soddisfazione di bisogni o "voglie", ma per alfabetizzarsi in merito al conoscere, al sentire, al comunicare, (...) in termini di rispetto e pace» (Contini, 2016, p. 40).

L'infanzia è competente, come diceva qualcuno, ma questa sua competenza, per dirla con Montessori, va "aiutata"... ad esprimersi, ad emergere, ad essere perseguita anche a scapito di qualche tempo "vuoto", di momenti di noia, di spazi in cui sperimentare la forza dell'attesa e, perché no, la fatica della frustrazione. Affinché questo emerga e, al contempo, venga contenuto, il mondo adulto deve essere preparato a fare qualche passo indietro rispetto alle sue aspettative, ma a fare qualche sforzo in più nel suo "inclinarsi" a misura di bambino e, allo stesso tempo, a guardare oltre quello che appare per mettere in campo il sentimento del rispetto e la tutela del suo diritto di espressione. È faticoso ascoltare i bambini, scriveva Korczak, non tanto perché bisogna «mettersi al loro livello, abbassarsi, inclinarsi, farsi piccoli», piuttosto perché è necessario «innalzarsi» fino all'altezza delle loro idee e dei loro sentimenti, tirandosi, allungandosi e alzandosi sulla punta dei piedi (Korczak, 2011).

Per chiudere, un monito: la storia dell'infanzia ci insegna che se i bambini vengono trattati come piccoli adulti, loro si impegnano ad agire ed essere come tali. Ma non senza fatica, fisica e psichica, per adeguarsi ai ritmi, ai tempi, ai luoghi che vengono loro imposti, per salire su uno "sgabello" troppo alto, che non è stato regolato alla loro altezza. Una casa, una scuola, una città che sono pronte ad abbassare quello sgabello e a garantire ai bambini i loro diritti di "essere piccoli" sono un posto migliore, anche per noi. L'incontro più autentico con l'infanzia è, infatti, una "palestra" di dialogo con la diversità che, certo, preoccupa e inizialmente spaventa, ma anche che, una volta "prese le misure", arricchisce il nostro repertorio esistenziale, affinandone dimensioni essenziali quali l'ascolto, il rispetto, la condivisione.

\section{Riferimenti bibliografici}

Ammaniti M. (2015). La famiglia adolescente. Roma-Bari: Laterza.

Ariés P. (1994). Padri e figli nell'Europa medievale e moderna. Roma-Bari: Laterza.

Bateson G. (1977). Verso un'ecologia della mente. Milano: Adelphi.

Bauman Z. (2002). Modernità liquida. Roma-Bari: Laterza.

Becchi E., a cura di (1982). Metafore d'infanzia. In Aut Aut, 191-192.

Beck U. (2000). I rischi della libertà. L'individuo nell'epoca della globalizzazione. Bologna: il Mulino.

Bernardini I. (2012). Bambini e basta. Milano: Mondadori.

Bertin G.M. (1977). Nietzsche: l'inattuale, idea pedagogica. Firenze: La Nuova Italia.

Bourdieu, P. (1997). Sulla televisione. Milano: Feltrinelli. 
Cambi F., Ulivieri S. (1999). Infanzia e violenza. Forme, terapie, interpretazioni. Firenze: La Nuova Italia.

Codeluppi V. (2007). La vetrinizzazione sociale: il processo di spettacolarizzazione degli individui e della società. Torino: Bollati Boringhieri.

Contini M., a cura di (2010). Molte infanzie molte famiglie. Roma: Carocci.

Contini M., Demozzi S. (2013). Corpi bambini. Sprechi di infanzie. Videodocumentario. IBC Movie.

Contini M., Demozzi S. (2016). Corpi bambini. Sprechi di infanzie: Sprechi di infanzie. Milano: FrancoAngeli.

Contini M., Demozzi S., a cura di (2016). Corpi bambini. Sprechi di infanzie. Milano: FrancoAngeli.

D’Amato M. (2014). Ci siamo persi i bambini: Perché l'infanzia scompare. Roma-Bari: Laterza.

Demozzi S. (2016). L’infanzia “inattuale”. Perché le bambine e i bambini hanno diritto al rispetto. Bergamo: Junior.

Demozzi S. (2016). L'infanzia inattuale. Bergamo: Junior.

Dolto F. (2005). I problemi dei bambini. Milano: Mondadori.

Ehrenberg A. (2010). La fatica di essere se stessi. Depressione e società. Torino: Einaudi.

Ferraris A.O. (2008). La sindrome Lolita: perché i nostri figli crescono troppo in fretta. Milano: Rizzoli.

Forni E., Altan F. (2005). La prospettiva del ranocchio: lo sguardo dei bambini sul mondo adulto. Torino: Bollati Boringhieri.

Galimberti U. (2002). Gli analfabeti delle emozioni. In La Repubblica.it. Testo disponibile al sito: http://www.repubblica.it/online/cronaca/desire/analfabeti/ analfabeti.html.

Galtung J. (1990). Cultural violence. In Journal of peace research, 27(3), 291-305.

Gigli A. (2016). Famiglie evolute. Capire e sostenere le funzioni educative delle famiglie plurali. Bergamo: Junior.

Kohan W. (2014). Childhood, Education and Philosophy: New Ideas for an Old Relationship. Abingdon: Taylor \& Francis.

Korczak J. (2011). Il diritto del bambino al rispetto. Bologna: Edizioni dell'Asino.

Lipman M. (2001). Stupirsi di fronte al mondo. Ragionare sulla natura. Napoli: Liguori.

Lorenzoni F. (2014). I bambini pensano grande. Cronaca di una avventura pedagogica. Palermo: Sellerio.

Miller A., (2010). Il dramma del bambino dotato e la ricerca del vero Sé. Riscrittura e continuazione. Torino: Bollati Boringhieri.

Morandi M., a cura di (2016). Corpo, educazione fisica, sport. Questioni pedagogiche. Milano: FrancoAngeli.

Morin E. (2000). La testa ben fatta. Riforma dell'insegnamento e riforma del pensiero. Milano: Raffaello Cortina.

Postman N. (2005). La scomparsa dell'infanzia. Roma: Armando.

Rilke R. M., (1995). Sonetti a Orfeo. Roma: Edizioni Studio Tesi.

Satta C. (2012). Bambini e adulti: la nuova sociologia dell'infanzia. Roma: Carocci.

Winnicott D.W. (1971). Gioco e realtà. Roma: Armando. 